\title{
Passenger Distribution and Waiting Position Selection Model on Metro Station Platform
}

\author{
Zongjie Liü, a, Dewei Li ${ }^{2, b}$ and Xiaoquan Wang ${ }^{3, c}$ \\ Department of Traffic and Transportation, Beijing Jiaotong University \\ No.3 Shang Yuan Cun, Hai Dian District, Beijing, China.100044

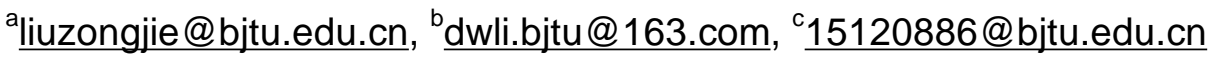

\begin{abstract}
Keywords: Metro station platform, waiting position selection, passenger distribution, dwell time Abstract: It is very important to obtain the distribution of passengers awaiting the train, which partly determines the number of passengers in each car and the train dwell time. The aim of this paper is to study and model the dynamic distribution of passengers on metro station platforms prior to a train's arrival. First, in the paper we analyze and summarize the primary influencing factors on the position selection for passengers to await the train. Second, a position selection model for each passenger based on an utility function was developed. It is solved by using a successive distribution approach, which can express the waiting position selection process of each passenger before the arrival of a train. To improve the accuracy, an alternating fitting-approaching one by one method is used to calibrate the model. Finally, the model is validated by applying to an actual case. A field survey on Pinganli Station of Beijing Subway Line 4 was conducted. The distribution data during evening peak and off-peak hours was collected. The application result has shown that the fitting result during the evening peak is more effective than the off-peak. We conclude that this model has expressed well the waiting position selection process of each passenger before the arrival of a train, which proves the model has good effectiveness and applicability.
\end{abstract}

\section{Introduction}

The distribution of passengers waiting on the platform (DPW) before the arrival of a train is one of the most important factors in optimizing the metro station design.

In the ideal situation, the DPW was usually assumed to be uniform to determine the size of the platform. However, Szplett and Wirasinghe [1] observed that the passenger distribution was not uniformly and closely related to the positions of platform access points. Lam et al. [2] considered that in different periods, the levels of service of different waiting positions were different, which reflected the non-uniformity of the passenger distribution. Wiggenraad [3] found that the passenger waiting distribution was closely related to the positions of platform access points, but there were made no further detailed studies. To study the formation mechanism of the non-uniform passenger distribution, Kim et al. [4] conducted a questionnaire survey of the reasons why a lot of passengers in the morning peak selected specific waiting positions on the platform. Krstanoski [5] regarded that multinomial probability distribution appeared to be a good model to describe the DPW. Based on the factors analyzed, Cao [6] established an attraction model to predict the DPW prior to the arrival of a train. Song et al. [7] developed stochastic user equilibrium model based on the utility function.

Although many studies developed various models to interpret the non-uniform passenger distribution, few of them could clarify the position selection process. So the research objective of this study is to develop a model which can express the waiting position selection process of each passenger before the arrival of a train.

Based on the literature review, section 2 summarizes the factors influencing DPW. Section 3 presents modelling expressing the waiting position selection process of each passenger before the arrival of a train. Section 4 calibrates the model. Section 5 applies the founded model to an actual case. Section 6 summarizes the research results. 


\section{Influencing Factors}

Different waiting position selections lead to the non-uniformity of the DPW. The selections are usually influenced by some factors which are listed as follows:

(1)The distance between a waiting position and a platform entrance

The waiting positions close to the platform entrance are selected by more passengers compared with the positions to which they need to spend more walking time.

(2)The number of passengers at the waiting position

Each waiting position has a fixed capacity and higher congestion degree will cause less attraction.

(3) The angle formed by a waiting position and the direction of the passenger flow

Cao [6] regarded that more passengers selected the waiting position whose angle between the directions of passengers pulling ranges from $15^{\circ}$ to $90^{\circ}$ (see $\alpha$ in Figure 1). In addition, a correlation exists between the distance and the angle factor [7].

(4)The exit location of the destination station

Kim [4] found that 69.7 percent of passengers who selected specific waiting positions were able to reduce the distance from alighting doors to the exits in the destination station.

(5)Guidance service level on the platform

The guidance in manual or broadcasting on the platform might lead passengers to the positions where it guides to.

(6)The layout of the platform facilities

Some facilities, such as pillars and guardrails which may hinder passengers queuing on the waiting area and influence the passenger's selection of waiting position.

\section{Waiting Position Selection Model}

Before developing the model, some assumptions are given as follows:

(1)The passengers who arrive after the train stop are not considered;

(2)All passengers know all waiting position situations immediately as soon as they reach the waiting area. After that they can select the most suitable position to await the train;

(3)All passengers will not wander on the platform after they have selected waiting positions. Their selections will not change when the train reaches the platform;

(4)When the number of passengers at a waiting position reaches capacity, the following passengers will not select this waiting position to queue up;

(5)The waiting positions are mutual independent. When a passenger selects a waiting position, the person will only consider the main factors to select this waiting position. At the same time, other waiting positions will not be influenced by this passenger's selection.

(6)The space of the platform is ample enough to avoid the congestion that make passengers be prevented from reaching waiting positions they select.

The value of utility is used to evaluate the waiting position's suitability for passengers. The value of utility of the passengers which come from entrance $j$ to waiting position $i$ is defined as $V_{i j}$. The number of passengers who have waited at waiting position $i$ is defined as independent variable $x_{i}$. The utility function $V_{i j}(x)$ is strictly decreasing with $x_{i}$. The limiting condition is as follow:

$$
\begin{aligned}
& \sum_{i=1}^{n} x_{i}=\sum_{j=1}^{m} q_{j} \times T \quad \forall i_{j} j \\
& 0 \leq x_{i} \leq c_{i} \quad \forall i_{n} j
\end{aligned}
$$

Where $c_{i}$ is the capacity of waiting position $i ; m$ is the number of the entrances; $n$ is the number of the waiting positions; $q_{j}$ is the passenger arrival rate of entrance $j ; T$ is the departing time interval.

The waiting position selection model is as follow: 


$$
\begin{aligned}
& \operatorname{Max} Z(x)=\sum_{i=1}^{n} \sum_{j=1}^{m} \sum_{x=0}^{x_{i}} V_{i j}(x) \\
& \text { S.T. } \sum_{i=1}^{n} x_{i}=\sum_{j=1}^{m} q_{j} \times T \quad \forall i_{j} j \\
& 0 \leq x_{i} \leq c_{i} \quad \forall i_{j} j
\end{aligned}
$$

The objective function is developed to maximize the sum of all the passengers' value of utility. The strictly decreasing utility function, the mutual independence and assumption (2) ensure the maximum value of utility of each passenger.

The main factors which can be quantified are selected to develop the strictly decreasing utility function with $x_{i}$ :

$$
V_{i j}(x)=\frac{A_{i j} \times\left[1-\left(\frac{x_{i}}{c_{i}}\right)^{\beta}\right]}{t_{i j}^{2}}
$$

Where $t_{i j}$ is the time that passengers need to walk from entrance $j$ to waiting position $i$; $A_{i j}$ is the combined influence of the rest of factors. $A_{i j}$ and $\beta$ are parameters that need to be calibrated.

To express the waiting position selection process of each passenger before the arrival of a train, a successive distribution approach is used to solve the model:

(1) $N$ is the sum of the passengers at all the waiting positions before the arrival of a train. $d$ is the passenger arrival rate proportion of entrance 2 and entrance 1.

(2)The value of utility in each waiting position should be initialized. The passengers at the waiting position are cleared after the last train leaves and the value of $x_{i}$ is equal to zero. The value of utility $V_{i j}$ can be obtained.

(3) $l$ is the number of passengers from entrance 1 while $r$ is the number of passengers from entrance 2. The first passenger is defined to arrive from entrance 1 . The passenger will be distributed to the waiting position of which value of utility is largest, and adds one to both the number of passengers at this waiting position and the value of $l$. The new value of utility $V_{i j}$ can be obtained.

(4)If the value of the proportion of $r$ and $l$ is not less than $d$, the next passenger is defined to arrive from entrance 1 and distributed to the waiting position whose value of utility is largest, and adds one to both the number of passengers at this waiting position and the value of $l$. If the value of the proportion of $r$ and $l$ is less than $d$, the next passenger is defined to arrive from entrance 2 and distributed to the waiting position whose value of utility is largest, and adds one to both the number of passengers at this waiting position and the value of $r$. The new value of utility $V_{i j}$ can be obtained. If the sum of $r$ and $l$ is less than $N$, the step (4) should be repeated. The computation will end when the sum of $r$ and $l$ is equal to $N$.

\section{Model Calibration}

$\beta$ and $A_{i j}$ in the utility function for each entrance should be calibrated. An evaluation index is given to evaluate the fitting degree of the results:

$$
S=\frac{1}{K} \sum_{i=1}^{n}\left(x_{i e}-x_{i s}\right)^{2}
$$

Where $k$ is the number of the fitting data groups. $x_{i c}$ is the predicted number of passengers at waiting position $i . x_{i s}$ is the observed number of passengers at waiting position $i$. 
The model uses an alternating fitting-approaching one by one method based on the collected data to calibrate $\beta$ and $A_{i j}$. The specific steps are listed as follows:

(1)The value of $\beta$ of entrance 1 and entrance 2 is set to be equal to 3 . The average number of passengers at each waiting position among the collected data is set to be the initial fitting data. Assume that the value of utility of each waiting position is 1 after the distribution completes, then $A_{i j}$ for each entrance and each waiting position can be obtained. The obtained value is set as the initial value of $A_{i j}$.

(2)According to $\beta$ and $A_{i j}$, the model can be solved with all collected distribution data groups and the value of $S$ can be obtained by Equation (4).

(3)Adjust $\beta$ of entrance 1 to get the minimum of $S$. The best $\beta$ of entrance 1 can be obtained.

(4)According to the value of $\beta$, each $A_{i j}$ for entrance 1 will be adjusted step by step. If an $A_{i j}$ having been adjusted makes $S$ reduced, this $A_{i j}$ should be adjusted further until the minimum of $S$ is obtained. Each $A_{i j}$ can get the best value by the same method.

(5)Step (3) and step (4) should be repeated to get the best value of $\beta$ and $A_{i j}$ of entrance 2.

(6)Step (3), step (4) and step (5) should be repeated until $\beta$ and $A_{i j}$ of entrance 1 and entrance 2 can't be adjusted further, which means the end of the model calibration.

\section{Case Study}

We selected Pinganli Station platform of Beijing Subway Line 4 to conduct a field survey. Since the station is a transfer station, and the number of transfer passengers from Line 6 to Line 4 Tiangongyuan direction is moderate, which is convenient to collect data.

There are twenty four doors on one side of the train. There are two entrances to the platform: Entrance 1 and Entrance 2. Figure 1 illustrates the layout of the platform at Pinganli Station. The manual count method was used to record the number of waiting passengers at each position in Tiangongyuan direction during the evening peak from 17:00 to 19:00 and the off-peak from 11:30 to 13:30. Every time that a train came to a stop on the platform, investigators recorded the numbers.

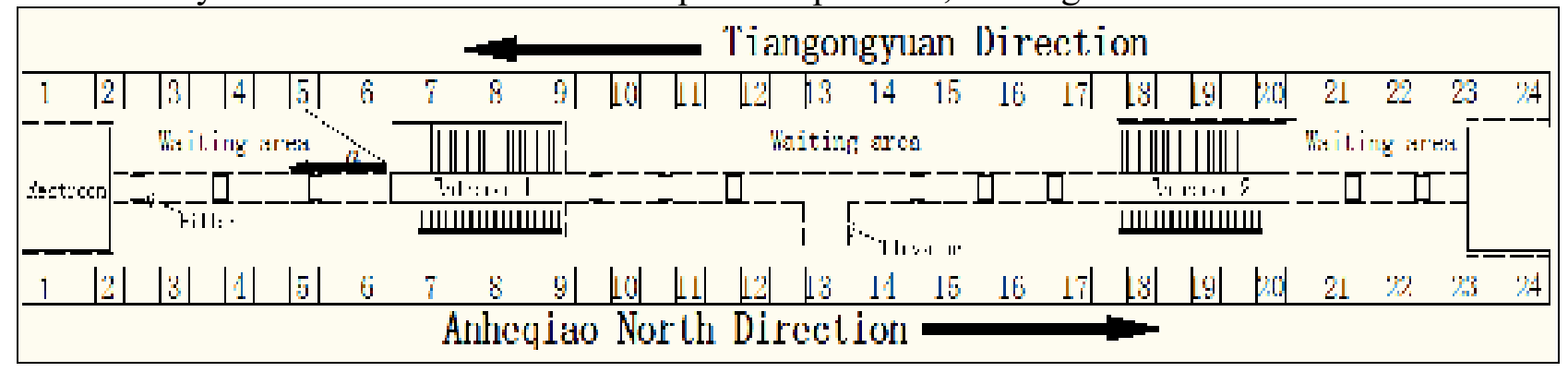

\section{FIGURE 1 Pinganli Station Platform Layout}

According to the field survey, $t_{i j}=l_{i j} / v \quad\left(l_{i j}\right.$ is the distance that passengers need to walk from entrance $j$ to waiting position $i$; $v$ is the free walking speed which is equal to $1.2 \mathrm{~m} / \mathrm{s}) ; c_{i}=S_{i} / D\left(S_{i}\right.$ is the area of waiting position $i ; D$ is per capita area which is equal to $0.206 \mathrm{~m}^{2}$ according to the collected data processing); $d=N_{2} / N_{l}\left(N_{l}\right.$ and $N_{2}$ are the numbers of passengers entering from entrance 1 and entrance 2 respectively). $l_{i j}, t_{i j}, c_{i} 、 S_{i} 、 N_{1} 、 N_{2}$ and $d$ could be worked out by processing the collected data. The case study results of model calibration are summarized in Table 1. 
TABLE 1 Model Calibration Results

\begin{tabular}{|c|c|c|c|c|c|c|c|}
\hline \multicolumn{4}{|c|}{ Evening Peak } & \multicolumn{4}{|c|}{ Off-Peak } \\
\hline \multicolumn{2}{|c|}{ Entrance 1} & \multicolumn{2}{|c|}{ Entrance 2} & \multicolumn{2}{|c|}{ Entrance 1} & \multicolumn{2}{|c|}{ Entrance 2} \\
\hline $\begin{array}{l}\text { Waiting } \\
\text { Position }\end{array}$ & $A_{i 1}$ & $\begin{array}{l}\text { Waiting } \\
\text { Position }\end{array}$ & $A_{i 2}$ & $\begin{array}{l}\text { Waiting } \\
\text { Position }\end{array}$ & $A_{i 1}$ & $\begin{array}{l}\text { Waiting } \\
\text { Position }\end{array}$ & $A_{i 2}$ \\
\hline 1 & 469.778 & 9 & 2121.34 & 1 & 429.004 & 9 & 1931.4 \\
\hline 2 & 335.52 & 10 & 1639.98 & 2 & 296.946 & 10 & 1612.17 \\
\hline 3 & 181.256 & 11 & 1333.23 & 3 & 176.75 & 11 & 1325.39 \\
\hline 4 & 90.3723 & 12 & 1074.48 & 4 & 87.8844 & 12 & 1064.85 \\
\hline 5 & 34.4215 & 13 & 780.61 & 5 & 33.1968 & 13 & 777.145 \\
\hline 6 & 5.94518 & 14 & 585.486 & 6 & 6.46729 & 14 & 583.216 \\
\hline 7 & 12.5851 & 15 & 418.976 & 7 & 12.0348 & 15 & 417.137 \\
\hline 8 & 48.6989 & 16 & 280.115 & 8 & 46.0078 & 16 & 279.058 \\
\hline 9 & 139.387 & 17 & 169.284 & 9 & 130.01 & 17 & 169.209 \\
\hline 10 & 227.222 & 18 & 85.6055 & 10 & 224.339 & 18 & 84.721 \\
\hline 11 & 352.335 & 19 & 32.9724 & 11 & 349.653 & 19 & 31.9779 \\
\hline 12 & 504.134 & 20 & 8.29512 & 12 & 502.297 & 20 & 7.9013 \\
\hline 13 & 739.89 & 21 & 12.7142 & 13 & 736.399 & 21 & 13.3354 \\
\hline 14 & 958.481 & 22 & 48.0598 & 14 & 953.19 & 22 & 48.5541 \\
\hline 15 & 1205.05 & 23 & 118.252 & 15 & 1198.1 & 23 & 112.18 \\
\hline 16 & 1480.19 & 24 & 215.599 & 16 & 1471.84 & 24 & 204.183 \\
\hline$\beta$ & 2.021 & $\beta$ & 2.426 & $\beta$ & 2.979 & $\beta$ & 2.667 \\
\hline$S$ & & 49.3294 & & $S$ & & 18.756 & \\
\hline
\end{tabular}

Three groups of observation data during the evening peak and the off-peak on May 19th in Tiangongyuan direction of Pinganli Station platform were extracted. Figure 2 shows the average value comparison between observations and predictions (left for the evening peak and right for the off-peak). Calculate the absolute value of each difference between the observation and the prediction. Table 2 shows the number and percentages of each absolute value during the evening peak and the off-peak.

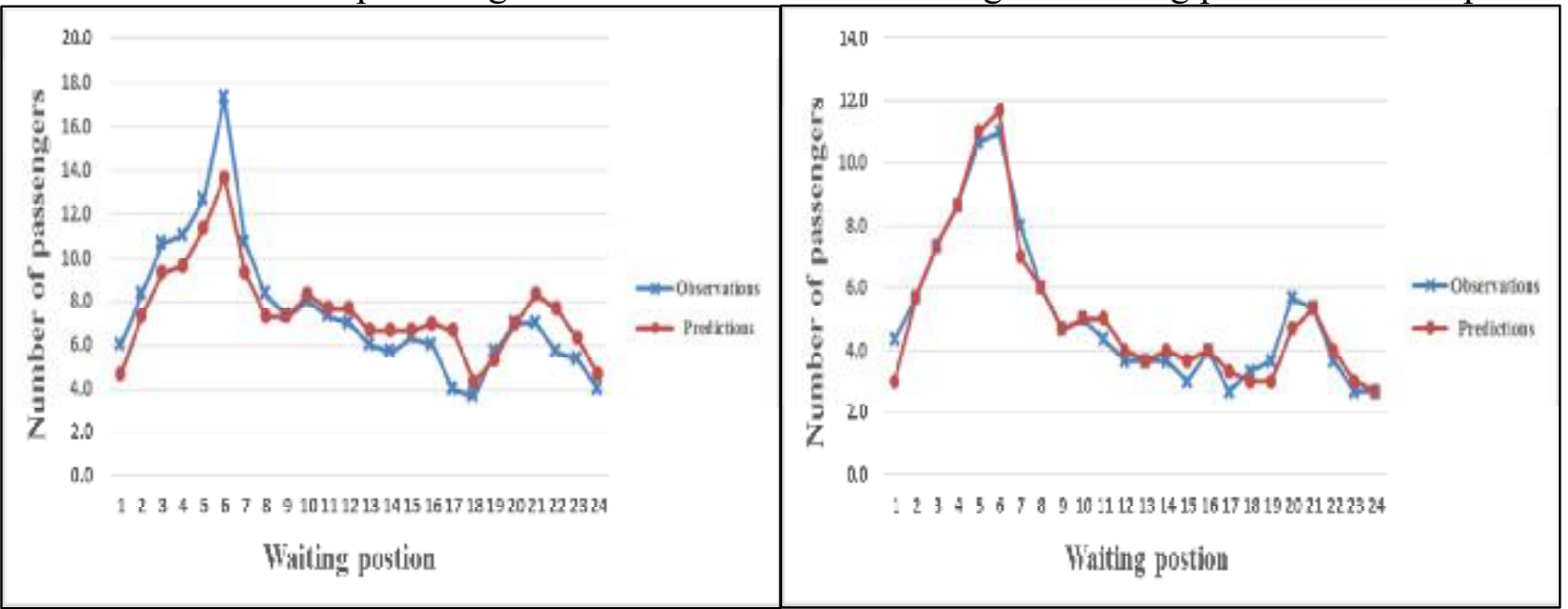

FIGURE 2 Average Value Comparison between Observations and Predictions 
TABLE 2 Percentages of Each Absolute Value

\begin{tabular}{ccc}
\hline $\begin{array}{c}\text { Absolute Value } \\
\text { of Error }\end{array}$ & $\begin{array}{c}\text { Evening Peak } \\
\text { Number/Percentage }\end{array}$ & $\begin{array}{c}\text { Off-Peak } \\
\text { Number/Percentage }\end{array}$ \\
\hline 0 & $19 / 26.39 \%$ & $30 / 41.67 \%$ \\
\hline 1 & $32 / 44.44 \%$ & $37 / 51.39 \%$ \\
\hline 2 & $14 / 19.44 \%$ & $4 / 5.56 \%$ \\
\hline 3 & $4 / 5.56 \%$ & $1 / 1.39 \%$ \\
\hline 4 & $2 / 2.78 \%$ & $0 / 0.00 \%$ \\
\hline 5 & $0 / 0.00 \%$ & $0 / 0.00 \%$ \\
\hline 6 & $1 / 1.39 \%$ & $0 / 0.00 \%$ \\
\hline
\end{tabular}

(1)According to Figure 2, the passenger distribution curves of observations and predictions are rather similar.

(2)According to Table 2, the maximum error during the evening peak and the off-peak are 6 and 3 passengers respectively. $26.39 \%$ and $41.67 \%$ of the absolute values of error are zero during the evening peak and the off-peak respectively. The percentage of the accumulated error which is equal or less than 2 is 90.28 during the evening peak and 98.61 during the off-peak. And according to Equation (4), $S$ is 64 during the evening peak and 20.7 during the off-peak.

In general, the model fitting result is more effective during the off-peak than during the evening peak. This position selection model can express well the waiting position selection process of each passenger before the arrival of a train which proves the model has good effectiveness and applicability.

\section{Conclusions}

In this paper, the analysis of influencing factors is discussed. A utility of waiting for trains is defined and a waiting position selection model is developed. A successive distribution approach is used to solve the model and an alternating fitting-approaching one by one method is used to calibrate the model. Based on the field survey data, the model is calibrated. The case study result shows that the model fitting result is more effective during the off-peak than during the evening peak. This position selection model can express well the waiting position selection process of each passenger before the arrival of a train, which proves the model has good effectiveness and applicability.

\section{Acknowledgements}

This research is supported by the Fundamental Research Funds for the Central Universities (2015JBM046), Beijing Natural Science Foundation (9132015), Beijing Higher Education Young Elite Teacher Project (YETP0555) and Fundamental Research Funds for the Central Universities (2014JBZ008).

\section{References}

[1] Szplett, D., Wirasinghe, S.C., An investigation of Passenger interchange and train standing time at LRT stations:(i) alighting, boarding and platform distribution of passengers[J].Journal of Advanced Transportation, 1984:18(1),1-12.

[2] Lam W H K, Cheung C Y, Lam C F, A study of crowding effects at the Hong Kong light rail transit stations[J].Transportation Research Part A: Policy and Practice, 1999, 33(5): 401-415.

[3] Wiggenraad P B L, Alighting and boarding times of passengers at Dutch railway stations[J].TRAIL Research School, Delft, 2001.

[4] Kim H, Kwon S, Wu S K, et al., Why do passengers select a specific car of a metro train during the morning peak hours[J].Transportation research part A: policy and practice, 2014, 61: 249-258. 
[5] Krstanoski N, Modelling passenger distribution on metro station platform[J].International Journal for Traffic \& Transport Engineering, 2014, 4(4).

[6] Cao, S.h., Analysis and Modelling on Passengers Traffic Characteristics for Urban Rail Transit. Beijing Jiaotong University, 2009.

[7] Song, Q.m., Wu, F., Yuan, Z.z., Analysis and Modeling of Passenger Distribution on Urban Rail Transit Platform [J].Urban Mass Transit, 2011, 09:43-47. 